\title{
Learning Behavior and Temporary Minima of Two-Layer Neural Networks
}

\author{
ANNE-Johan ANNEMA, KlaAs HoEN, AND HaNS Wallinga \\ MESA Research Institute, University of Twente
}

(Received 8 March 1993; accepted 18 March 1994)

\begin{abstract}
This paper presents a mathematical analysis of the occurrence of temporary minima during training of a single-output, two-layer neural network, with learning according to the back-propagation algorithm. A new vector decomposition method is introduced, which simplifies the mathematical analysis of learning of neural networks considerably. The analysis shows that temporary minima are inherent to multilayer networks learning. A number of numerical results illustrate the analytical conclusions.
\end{abstract}

Keywords-Neural networks, Multilayer perceptron, Learning, Temporary minimum, Back propagation, Pattern classification.

\section{INTRODUCTION}

Neural networks generally consist of a large number of simple processing elements and interconnections. The simple processing elements are called neurons. Every neuron has multiple input signals and one output signal. The output is typically a nonlinear function of the sum of the weighted input signals of that neuron.

A widely used neural network architecture is the multilayer feedforward structure (Lippmann, 1987). Such networks can be trained to perform a vector classification by presenting examples (Lippmann, 1987; Hornik, Stinchcombe, \& White, 1990; Irie \& Miyake, 1988 ). During training of a neural network, the weights of all neurons in the net are adapted according to a training algorithm. In the past few years, the interest in multilayer feedforward neural networks has grown considerably as a result of research into the development of efficient training algorithms for multilayer networks. In particular, the training algorithm presented by Rumelhart et al., the so-called back-propagation al-

\footnotetext{
Acknowledgements: The authors would like to thank R. J. Wiegerink, Department of Applied Physics, University of Twente, C. C. A. M. Gielen, University of Nijmegen, and P. I. M. Johannesma for fruitful discussions and useful comments. Furthermore, the unknown reviewer who challenged us to include the XOR function is acknowledged. These investigations in the program of the Foundation for Fundamental Research on Matter (FOM) have been supported by the Netherlands Technology Foundation (STW).

Requests for reprints should be sent to Anne-Johan Annema, MESA Research Institute, Twente University, P.O. Box 217, 7500AE Enschede, The Netherlands.
}

gorithm (Rumelhart, Hinton, \& Williams, 1986), is widely known.

Considerable research has been done in the field of multilayer neural networks with respect to feedforward network abilities (Akaho \& Amari, 1990; Arai, 1989; Huang \& Huang, 1991; Mehrotra, Mohan, \& Ranka, 1991; Heskes, Slijpen, \& Kappen, 1992). The dynamic behavior of a single-layer neural network has been well analyzed (Sontag \& Sussmann, 1991; Minsky \& Papert, 1988). However, the learning behavior or dynamics during training of a multilayer neural network is generally derived by simulations because mathematical analyses of dynamics of nonlinear systems, such as a multilayer neural network, are very complicated. Only recently, a mathematical analysis of the dynamics of a feedforward multilayer network has been published (Guo \& Gelfand, 1991). Although mathematical analyses may require drastic simplifications to derive easyto-read equations, they contribute much more to the understanding of the fundamental principles involved than do only simulations.

A major problem that occurs during multilayer perceptron learning is encountering undesired minima. In undesired minima, the performance improvement of the classification drops to very low levels or even approaches zero whereas the network has not (yet) arrived at the optimal state for classification of the training set. Two types of undesired minima can be distinguished, local minima (Hirose, Yamashita, \& Hijiya, 1991) and temporary minima (Murray, 1991a).

The performance improvement of the classification drops to zero if the network is getting stuck in a local minimum in the energy landscape. The local minimum 
may be abandoned by adding noise or by using sophisticated convergence algorithms. Possible modifications include simulated annealing (Sheu, 1991; Murray, 1991b) and applying another energy function (Hanson \& Burr, 1988).

Temporary minima (Murray, 1991a) are fundamentally different from local minima as the performance improvement in this type of minimum drops to a very low but nonzero level because an approximately flat region in the energy landscape is encountered. Without modifications to the training set or learning algorithm, the network may escape from this type of minimum, but performance improvement in these temporary minima is very low because of the very low gradient in the energy landscape. In the mean squared error (MSE) versus training time curve, a temporary minimum can be recognized as a phase in which the MSE is virtually constant for a long training time after initial learning. After a generally long training time, the approximately flat part in the energy landscape is abandoned, resulting in a significant and sudden drop in the MSE curve (Murray, 1991a; Woods, 1988).

As temporary minima slow down learning significantly, minimizing the training time that is spent in this type of undesired minima speeds up learning considerably. In this article, the fundamental process behind the temporary minima is derived mathematically under condition of small initial weights. This condition will generally be satisfied in neural network training if no a priori knowledge about the training set is included in initial weights. The applicability of the presented analysis if the condition of small initial weights is not fulfilled is discussed.

The outline of this paper is as follows. A short introduction into the back-propagation training algorithm and into the used notation is given in Section 2. The vector decomposition method used for the analysis of the fundamental process behind the temporary minima during learning is introduced in Section 3.

The initial training and assumptions used for the analyses in this paper are discussed in the first part of Section 4. The remainder of Section 4 presents an introduction into the analyses of two (strongly related) mechanisms, called the "rotation-based" and "translation-based" mechanisms, that lead towards the encounter of temporary minima.

The rotation-based mechanism is analyzed in Section 5; an extensive discussion of this mechanism is included in this section. An illustrative example of this mechanism is presented in Section 6.

The analysis and discussion of the translation-based mechanism are presented in Section 7. As the analysis of this mechanism is strongly related to that of the rotation-based mechanism, the analysis and discussions are kept short. In Section 8, the XOR problem is selected to briefly illustrate the conclusions of Section 7 .
The analyses in Sections 4-8 will be done for a twolayer network with two neurons in the first layer. An extension towards neural networks with an arbitrary number of neurons in the first layer is given in Section 9. Section 10 summarizes the conclusions.

\section{NETWORK DEFINITION AND BACK-PROPAGATION LEARNING}

In this article, the dynamics of a single-output twolayer neural network during training are analyzed. The learning algorithm is the back-propagation rule.

A general form for a single-output, two-layer neural network is given in Figure 1. The two-layer neural network has $N_{\text {in }}$ external input signals and one bias input. Note that the bias signals are identical for all neurons in the network. The first layer contains $N_{1}$ neurons and the second layer, which is the output layer, contains one neuron.

The vector containing all input signals of the neurons in the first layer is called the input vector of the network

$$
\underline{U}_{1}=\left(U_{1}, U_{2}, \ldots, U_{N_{\text {in }}}, U_{\text {bias }}\right)^{T} .
$$

The input vector of the neuron in the second layer is

$$
\underline{U}_{2}=\left(Y_{1,1}, Y_{1,2}, \ldots, Y_{1, N_{1}}, U_{\text {bias }}\right)^{T},
$$

where $Y_{k, j}$ denotes the output of the $j$ th neuron in the $k$ th layer. A neuron weights every input signal and performs a nonlinear function $f(\cdot)$ on the weighted input. The weights of the $j$ th neuron in the $k$ th layer form a weight vector $W_{k, j}$. The response of a neuron is

$$
Y_{k, j}=f\left(\underline{W}_{k, j} \cdot \underline{U}_{k}\right) .
$$

The response of the neural network is the response of the neuron in the second layer,

$$
Y_{\text {net }}=Y_{2,1}=f\left(\underline{W}_{2,1} \cdot \underline{U}_{2}\right) .
$$

During training of the neural network, input vectors $U_{1}^{p}$ and the associated desired response of the network $D^{p}$ for every training vector are presented. For every training vector, the learning algorithm will adapt the weight vectors of all neurons to minimize a predefined energy function.

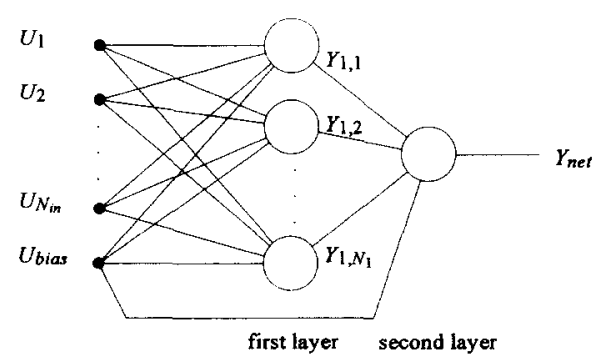

FIGURE 1. General single-output, two-layer feedfonward neural network. 
In this article, we assume that the two-layer neural network is trained using the back-propagation learning algorithm (Rumelhart, Hinton, \& Williams, 1986). The back-propagation rule is probably the most widely used learning algorithm for multilayer feedforward neural networks. It was discovered by Werbos in 1974 (Werbos, 1988), rediscovered by Parker in 1982 (Parker, 1985), and again rediscovered by Rumelhart et al. in 1986 (Rumelhart et al., 1986).

The back-propagation learning algorithm is based on the minimization of the summed squared error between the actual responses $Y_{2,1}^{p}$ and the associated desired responses $D^{p}$ of the network over all training examples. Hence, the energy function

$$
E=\frac{1}{2} \sum_{p}\left(D^{p}-Y_{2,1}^{p}\right)^{2}=\sum_{p} E^{p}
$$

is to be minimized during training. This minimization is done using a gradient descent minimization method, resulting in

$$
\Delta \underline{W}_{k, j}=-\eta \frac{\partial E}{\partial \underline{W}_{k, j}}=-\eta \sum_{p} \frac{\partial E^{p}}{\partial \underline{W}_{k, j}},
$$

with $\eta$ a small, positive constant. In a frequently used approximation of eqn (6), the weight vectors of the neurons in the neural network are adapted after presentation of any training example. This "local learning" approximation is allowed for small learning rates $\eta$ (Rumelhart et al., 1986). In the analysis, we use the original back-propagation algorithm given by eqn (6); that is, batch learning or global learning is used. However, the results obtained hold as a good approximation as well for the local learning back-propagation algorithm.

\section{VECTOR DECOMPOSITION}

For the analysis of the dynamics during training of a two-layer neural network, we introduce a vector decomposition method. In this vector decomposition, the weight vectors and input vectors of all neurons in the network are decomposed into three vector components that are related to so-called attractor hyperplanes, to be introduced in this section. Every neuron in the network has its specific attractor hyperplane.

First, the attractor hyperplane will be introduced. The last part of this section deals with the decomposition of the weight vectors and input vectors of the neurons in the neural network.

\subsection{Hyperplane}

In a back-propagation neural net trained for pattern classification, the weight vector of a neuron corresponds to a hyperplane that separates the input space of the neuron into two classes (Lippmann, 1987; Yang \&
Guest, 1990; Liang, 1991). The equation of the hyperplane corresponding to a weight vector $\underline{W}_{k, j}$ is

$$
\underline{W}_{k, j} \cdot \underline{U}_{k}=0 \text {. }
$$

\subsection{Attractor Hyperplane}

A multilayer neural network is known to be a universal approximator (Hornik et al., 1990). Consequently, the weight vectors of the neurons in the network converge during training towards specific attractors in weight space (Guo \& Gelfand, 1991; Parker, 1985). The positions of these attractors in weight space are independent of the order in which the training examples are presented because global training is used. The actual attractors may depend on the initial weights. For reasons of conceptual simplicity, we assume the initial weights to be very small. The condition for very small initial weights will be defined in Section 4.

During training, several distinguishable phases will be surpassed. In each of the subsequent training phases, the attractors may be different from the previous attractor positions. This will become clear along with the analyses that follow in Sections 4-9. At this point, we like to ask the reader to keep up with our analysis, starting with the assumption of very small initial weights. The analysis will take the following line.

In the first phase of training using very small initial weights, the neural network can be linearized because all neurons are activated in the approximately linear middle region. In this phase, redundancy is built up in the first layer; in other words, the attractors in weight space for all neurons in the first layer are identical. After this phase, the first layer is approximately reducible to one neuron (Sussmann, 1992) and the weight vectors of the neurons in the first layer are approximately identical.

In the next phase, the network cannot be linearized any more and the network nonlinearities must be included in the analysis. In this phase, the cluster of redundant neurons in the first layer will first continue building up redundancy and may then start to abolish this redundancy partially by starting to break into two clusters of neurons. During this phase, the attractors in weight space are still identical for all neurons in the first layer.

A third phase continues the breaking of the cluster into two smaller clusters. If the cluster is effectively broken into two clusters, the weight vector of the neurons in each cluster now converge towards two significantly different attractors in weight space.

The attractor in weight space for a specific neuron will be denoted as the weight vector attractor, $\underline{W}_{k, j}^{\mathrm{ATT}}$. Because of the different phases that will be shown to be surpassed during training in Sections 4, 5, 7, and 9, the weight vector attractor will generally be different in different phases. The hyperplane corresponding to the 
weight vector attractor will be denoted as the attractor hyperplane and will henceforth generally be different in different phases during training.

\subsection{Vector Decomposition}

For the analysis, we propose to decompose the weight vector and the input vector of every neuron in the neural network into three orthogonal vector components related to its attractor hyperplane. One of the vector components is related to only the bias input of the neurons, one component is perpendicular to the attractor hyperplane, and the last vector component is in parallel to the attractor hyperplane of the corresponding neuron.

The decomposition is best illustrated in two steps. The first step is to decompose both the weight and input vector of a neuron into two vectors, one vector component related to only the bias input signal as defined in Section 2 and denoted by the superscript bias, and the other vector component related to all nonbias-related input signals and denoted by the superscript $r$. The result of this first step is

$$
\underline{U}_{k}=\underline{U}_{k, j}^{\text {bias }}+\underline{U}_{k, j}^{r}
$$

and

$$
\underline{W}_{k, j}=\underline{W}_{k, j}^{\text {bias }}+\underline{W}_{k, j}^{r} .
$$

For the vector components in eqns ( 8 ) and (9) hold:

$$
\begin{gathered}
\underline{U}_{k, j}^{r} \perp \underline{U}_{k, j}^{\text {bias }}, \\
\underline{W}_{k, j}^{r} \perp \underline{W}_{k, j}^{\text {bias }},
\end{gathered}
$$

and

$$
\underline{U}_{k, j}^{\text {bias }} / / \underline{W}_{k, j}^{\text {bias }} \text {. }
$$

The nonbias-related vector components $\underline{U}_{k, j}^{r}$ and $\underline{W}_{k, j}^{r}$ are now decomposed into two orthogonal vector components, respectively perpendicular and in parallel to the attractor hyperplane. The weight vector and input vector component perpendicular to the attractor hyperplane will be denoted $\underline{W}^{h}$ and $\underline{U}^{h}$. These two vector components satisfy

$$
\underline{W}_{k, j}^{\mathrm{ATT}} \cdot \underline{U}_{k, j}^{r}=\underline{\underline{\mathrm{ATT}}} \cdot \underline{U}_{k, j}^{h}
$$

and

$$
\underline{W}_{k, j}^{\mathrm{ArT}} \cdot \underline{W}_{k, j}^{r}=\underline{W}_{k, j}^{\mathrm{ATT}} \cdot \underline{W}_{k, j}^{h} .
$$

The other vector components resulting from the decomposition are perpendicular to both the bias-related vector components and perpendicular to the weight vector attractor. For the input vector, this component is called $\underline{U}^{F}$, with

$$
\underline{U}_{k, j}^{F}=\underline{U}_{k, j}^{r}-\underline{U}_{k, j}^{h} .
$$

For the weight vector, this vector component is denoted as $\underline{W}^{e}$, with

$$
\underline{W}_{k, j}^{c}=\underline{W}_{k, j}^{r}-\underline{W}_{k, j}^{h} .
$$

Henceforth, the input vector and the weight vector of a neuron in a neural network are decomposed into the sets given in the following expressions:

$$
\begin{gathered}
\underline{U}_{k, j}=\underline{U}_{k, j}^{h}+\underline{U}_{k, j}^{\text {bias }}+\underline{U}_{k, j}^{k} \\
\underline{W}_{k, j}=\underline{W}_{k, j}^{h}+\underline{W}_{k, j}^{\text {bias }}+\underline{W}_{k, j}^{c} .
\end{gathered}
$$

\subsection{Quantification of the Vector Components}

Quantification of the vector components simplifies the notation in the vector decomposition-based analysis method. A simple quantification of the first two components of eqns (14) and (15) can be made by introducing two unity vectors,

$$
\underline{B}_{k, j}^{h}=\frac{\underline{U}_{k, j}^{h}}{\left|\underline{U}_{k, j}^{h}\right|}
$$

and

$$
\underline{B}_{k, j}^{\text {bias }}=\frac{\underline{U}_{k, j}^{\text {bias }}}{\left|\underline{U}_{k, j}^{\text {bias }}\right|} .
$$

The norms of the vector components in the $\underline{B}_{k, j}^{h}$ direction are represented by $\alpha_{k, j}^{h}$ for the input vector and by $\beta_{k, j}^{h}$ for the weight vector, and the norms of the vector component in the $B_{k, j}^{\text {bias }}$ direction are represented by $\alpha_{k, j}^{\text {bias }}$ for the input vector and by $\beta_{k, j}^{\text {bias }}$ for the weight vector. Quantification of the $\underline{U}_{k, j}^{F}$ and $\underline{W}_{k, j}^{i}$ vector components using unit vectors is not relevant within the scope of the present analysis. In this notation, ${ }^{1}$ we obtain

$$
\underline{U}_{k, j}=\alpha_{k, j}^{h} \underline{B}_{k, j}^{h}+\alpha_{k, j}^{\text {bias }} \underline{B}_{k, j}^{\text {bias }}+\underline{U}_{k, j}^{F}
$$

and

$$
\underline{W}_{k, j}=\beta_{k, j}^{h} \underline{B}_{k, j}^{h}+\beta_{k, j}^{\text {bias }} B_{k, j}^{\text {bias }}+\underline{W}_{k, j}^{c} .
$$

Figure 2 illustrates the decomposition of the weight vector of a neuron; the bias vector component is not shown in the figure. The shaded areas in Figure 2 mark the two classes to be separated by the neuron.

The response of a neuron is a nonlinear function of the weighted input of the neuron [eqn (3)]. With eqns (18) and (19), the response of a neuron is

$$
\begin{aligned}
Y_{k, j} & =f\left(\underline{W}_{k, j} \cdot \underline{U}_{k}\right) \\
& =f\left(\alpha_{k, j}^{h} \beta_{k, j}^{h}+\alpha_{k, j}^{\text {bias }} \beta_{k, j}^{\text {bias }}+U_{k, j}^{F} \cdot \underline{W}_{k, j}^{k}\right) .
\end{aligned}
$$

In the vector decomposition-based analyses, vector decompositions as shown in eqns (18) and (19), and neuron responses according to eqn (20) will be used.

\footnotetext{
${ }^{1}$ The $\alpha_{k, j}^{h}$ and $\beta_{k, j}^{h}$ may be associated with the concepts of relevant information and correct knowledge, respectively. Similarly, $\left|U_{k, j}, \boldsymbol{F}\right|$ and $\left|W_{k, j}{ }^{*}\right|$ may be associated with irrelevant information and incorrect knowledge, respectively.
} 


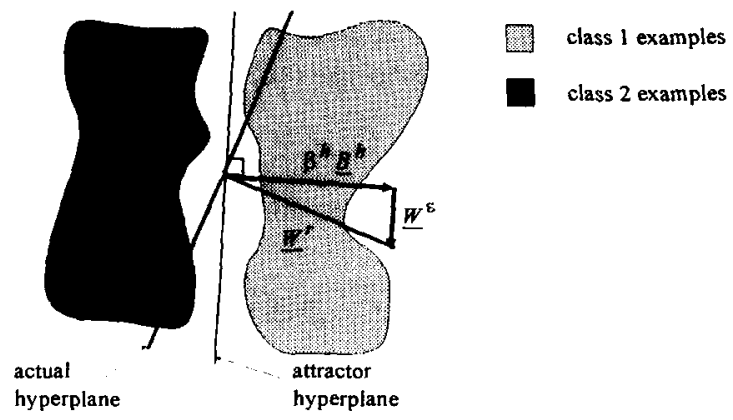

FIGURE 2. Weight vector decomposition; bias component not shown.

\section{ANALYSIS OF TEMPORARY MINIMA; INTRODUCTION}

The vector decomposition method will now be used to analyse the dynamic behavior of a learning neural network when, after initial training, a temporary minimum is encountered. In a temporary minimum, the energy landscape is approximately flat and hence the MSE is approximately constant for a relatively long training time. It is observed that, after a long training time, the MSE suddenly drops to a significantly lower level. Encountering a temporary minimum slows down the learning process significantly. Consequently, reducing the time during which the network sticks in this type of minima would speed up learning significantly. In this section, first a description of the learning behavior of two-layer neural networks in the very beginning of training is presented. For this description, it is assumed that the initial weights are small. The condition for small initial weights is usually satisfied in neural network training when no a priori knowledge about the training set is included in initial weights.

After this description, a short introduction of two related mechanisms (rotation-based and translationbased) that lead towards the encounter of temporary minima is presented. The rotation-based mechanism is analyzed in detail in Section 5, and the translationbased mechanism is analyzed in Section 7. Reduction of sticking time in temporary minima will be the subject of a future paper.

\subsection{Assumptions}

To obtain easy-to-read equations, we make some assumptions concerning the weight vectors of the neurons in the network and concerning the number of neurons in the network. These assumptions do not degenerate the generality of this approach.

Firstly, it is assumed that the number of neurons in the first layer of the network is two. In Section 9, it will be shown by induction that the analyses presented in Sections 4-7 hold for any single-output, two-layer network with an arbitrary number of neurons in the first layer.
A second assumption is that the initial weights of the neurons in the network are so small that all neurons are activated in the approximately linear middle region of the transfer function during the beginning of training. Initial weights satisfying this assumption are very small initial weights. As long as the weights are very small, the neural network can be linearized. Initial weights that are not very small are discussed in Section 9.

\subsection{Initial Training: A Linearized Network}

At the start of training, the weights of the neurons in the first layer are very small. The weighted input of neurons in the first layer will then be close to zero for any training example, $\underline{W}_{1, j} \cdot \underline{U}_{1}^{p} \approx 0$. Consequently, the response of any neuron in the first layer on any training example is approximately $f(0)$.

The adaptation of a weight of the neuron in the second layer for any single training example is given by

$$
\Delta \underline{W}_{2,1}[n]=\eta(D-Y) f^{\prime}\left(\underline{W}_{2,1} \cdot \underline{U}_{2}\right) \underline{U}_{2}[n] .
$$

The first three factors on the right-hand side of this equation are shared for all weights of the neuron. Only the last factor, $\underline{U}_{2}[n]$, is different for the update of different elements in $\underline{W}_{2,1}$. In the previous paragraph, it has been shown that for very small initial weights, the responses of the neurons in the first layer are closely identical. The weights in $\underline{w}_{2,1}$ connected to neurons in the first layer will therefore adapt identically on any training example in the very beginning of training.

The adaptation of the weight vectors of the neurons in the first layer is given by

$$
\Delta \underline{W}_{1, j}=\eta(D-Y) f^{\prime}\left(\underline{W}_{2,1} \cdot \underline{U}_{2}\right) \underline{W}_{2,1}[j] f^{\prime}\left(\underline{W}_{1, j} \cdot \underline{U}_{1}\right) \underline{U}_{1} .
$$

The first three factors on the right-hand side on this equation are again identical for all neurons in the first layer. Furthermore, as the elements in $\underline{W}_{2,1}$ corresponding to neurons in the first layer adapt identically on any training example, the weights $\underline{W}_{2,1}[j]$ are equal. The fifth factor on the right-hand side of the equation, $f^{\prime}\left(\underline{W}_{1, j} \cdot \underline{U}_{1}\right)$, is identical for all neurons assuming either very small weights or almost identical weight vectors $\underline{W}_{1, j}$. The input vector for the first layer $\underline{U}_{1}$ is finally fed to all neurons in the first layer and is hence identical for all neurons in the first layer.

Therefore, it can be concluded that in the beginning of training, the weight vectors $\underline{W}_{1, j}$ adapt by approximation identically on any training example and that furthermore the associated weights $\underline{W}_{2,1}[j]$ adapt closely identical. For global training, the weights are updated after presentation of the total training set. The weight updates for $\underline{W}_{2,1}[j]$ and the weight vector adaptations $\Delta \underline{W}_{1, j}$ are approximately equal for any training example. Consequently, in the case of global learning, the summed weight updates over the total training set are also closely identical for the neurons in the first layer and for the weights in $W_{2,1}$ associated with the output 
of these neurons. An illustration of the process of closely identical weight adaptation in the beginning of training is presented in Figure 4 in section 6 . The figure presents the dynamics of weights in weight space for the training set of Figure 3 in Section 6, as simulated by a neural network simulation program. In the beginning of training, that is, for $\underline{W}_{k, j}[n]$ small, the adaptation of $W_{1, j}^{r}$ appears to proceed almost identically. The same holds for the corresponding weights of the neuron in the second layer, $\underline{W}_{2,1}[j]$.

Note that there is a possibility that the weight vectors of the neurons in the first layer adapt in opposite directions, which introduces opposite signs for the associated weights of the neuron in the second layer. This situation will be discussed later on in this paper.

First we will analyse the learning behavior using the vector decomposition method. In the very beginning of the training and under condition of very small initial weights, the weight vectors of the neurons in the first layer adapt closely identically. The corresponding hyperplanes of the neurons in the first layer therefore move towards the same position in input space. Moving towards identical positions in input space of the hyperplanes can be interpreted as coinciding attractor hyperplanes for the two neurons in the first layer of the network:

$$
\underline{B}_{1,1}^{h}=\underline{B}_{1,2}^{h} \equiv \underline{B}_{1}^{h} .
$$

As the weight vectors of the two neurons in the first layer adapt almost identical and with eqn (21), the $\beta_{1, j}^{h}$ of the two neurons in the first layer adapt to approximately identical values as long as the network can be linearized. In the analysis, it is assumed for reasons of simplicity that

$$
\beta_{1,2}^{h}=\beta_{1,2}^{h} \equiv \beta_{1}^{h} .
$$

The weight vectors of the neurons in the first layer will, under condition of proper initialization, converge towards the same weight vector attractor. Based on the analysis of the dynamics of the neural network using very small initial weights, that is, weights that allow linearization of the network in early training, we posit the following theorem.

THEOREM 1. On condition of very small initial weights, the hyperplanes of the neurons in the first layer of a single-output, two-layer neural network will, in the first part of training, move towards identical positions in input space.

However, the weight vectors will not be exactly identical; they will generally have different $\underline{W}_{k, j}^{i}$ and $\beta_{k, j}^{\text {bias }}$ vector components. Due to the fact that the neural network can be linearized, the classification made by the network in the very beginning of training corresponds to the classification made by the average position of the hyperplanes of the neurons in the first layer. Because the weight vectors of both neurons in the first layer attract to their weight vector attractors $\underline{W}_{1}^{\mathrm{ATT}}$,

$$
\frac{1}{2}\left(\underline{W}_{1,1}+\underline{W}_{1,2}\right)=\underline{W}_{1,1}^{\mathrm{ATT}}=\underline{W}_{1,2}^{\mathrm{ATT}}=\underline{W}_{1}^{\mathrm{ATT}} .
$$

With the vector decomposition method, the $\underline{W}_{1}^{\mathrm{ATT}}$ is decomposed into

$$
\underline{W}_{1}^{\mathrm{ATT}}=\beta_{1}^{h} \underline{B}_{1}^{h}+\beta_{\mathrm{ATT}}^{\text {bias }} \underline{B}_{1}^{\text {bias }} .
$$

It follows from eqns (23) and (24) that as long as the network can be linearized, eqn (25) will be satisfied by good approximation:

$$
\left\{\begin{array}{l}
\underline{W}_{1,1}^{c}=-W_{1,2}^{e} \\
\beta_{1,1}^{\text {bias }}+\beta_{1,2}^{\text {bias }}=\beta_{\mathrm{ATT}}^{\text {bias }}
\end{array} .\right.
$$

For simplicity of presentation, both a vector $\Lambda W^{\mathrm{e}}$ denoting the difference of the $\underline{W}_{i, j}$ vectors and a differential norm of the $B_{1, j}^{\text {bias }}$ components are introduced. These new components satisfy:

$$
\left\{\begin{array}{l}
\Lambda W^{e}=W_{1,1}^{r}-W_{1,2}^{f} \\
\Lambda \beta_{1}^{\text {bias }}=\beta_{1,1}^{\text {bias }}-\beta_{1,2}^{\text {bias }}
\end{array}\right.
$$

It follows that when starting with very small initial weights that allow linearization of the network in the beginning of training, the weight vectors of the two neurons in the first layer adapt to

$$
\begin{aligned}
& \underline{W}_{1,1}=\beta_{1}^{h} \underline{B}_{1}^{h}+\left(\beta_{1}^{\text {bias }}+\frac{1}{2} \Lambda \beta_{1}^{\text {bias }}\right) \underline{B}_{1}^{\text {bias }}+\frac{1}{2} \underline{\Lambda} \underline{W}^{c} \\
& \underline{W}_{1.2}=\beta_{1}^{h} \underline{B}_{1}^{h}+\left(\beta_{1}^{\text {bias }}-\frac{1}{2} \Lambda \beta_{1}^{\text {bias }}\right) \underline{B}_{1}^{\text {bias }}-\frac{1}{2} \underline{\Lambda} \underline{W}^{c} .
\end{aligned}
$$

in the beginning of training.

It was also shown that the weights of the neuron in the second layer associated with neurons in the first layer, $\underline{W}_{2,1}[n]$, adapt (by approximation) identically in the beginning of training. It will first be assumed that the signs of these weights are equal; a discussion of a more general case will be given later on in this article.

$$
\underline{W}_{2,1}[n]=w_{2,1} \quad n \in\left\{1, \ldots, N_{1}\right\} \quad \text { with } N_{1}=2 .
$$

With eqn (28), the $\underline{B}_{2,1}^{h}$ vector is

$$
\underline{B}_{2,1}^{h}=\left[\begin{array}{c}
\sqrt{1 / N_{1}} \\
\sqrt{1 / N_{1}} \\
0
\end{array}\right]=\left[\begin{array}{c}
\sqrt{0.5} \\
\sqrt{0.5} \\
0
\end{array}\right] .
$$

\subsection{Continued Training: Including Network Nonlinearities}

The analysis of the learning behavior of two-layer networks as presented assumes that the weight vectors of the neurons in the first layer are close enough to satisfy condition (30):

$$
f\left(\underline{W}_{1,1} \cdot \underline{U}_{1}\right)+f\left(\underline{W}_{1,2} \cdot \underline{U}_{1}\right) \approx 2 f\left(\underline{W}_{\hat{\imath}}^{\mathrm{ATT}} \cdot \underline{U}_{1}\right) .
$$


With eqn (30), the response of the two-layer neural network on an input vector $\underline{U}_{1}$ is

$$
\begin{aligned}
Y\left(U_{1}\right)=f\left(\underline{W}_{2,1}[1] f\left(\underline{W}_{1,1} \cdot \underline{U}_{1}\right)\right. & \\
& \left.+\underline{W}_{2,1}[2] f\left(\underline{W}_{1,2} \cdot \underline{U}_{1}\right)+\alpha_{2,1}^{\text {bias }} \beta_{2,1}^{\text {bias }}\right) .
\end{aligned}
$$

With eqns (27) $-(30)$, this equation can be approximated by

$$
Y\left(U_{1}\right)=f\left(\sqrt{2} \beta_{2,1}^{h} f\left(\underline{W}_{1}^{\mathrm{ATT}} \cdot \underline{U}_{1}\right)+\alpha_{2,1}^{\text {bias }} \beta_{2,1}^{\text {bias }}\right) .
$$

The factor $\sqrt{2}$ in the first term of the argument springs from $N_{1} \sqrt{1 / N_{1}}=\sqrt{2}$ for a neural net with two neurons in the first layer (i.e., $N_{1}=2$ ).

Condition (30) includes eqn (23), but is valid in a larger range: condition (30) is not only satisfied for small weights (i.e., weights that allow linearization), but is also satisfied when the weight vectors $\underline{W}_{1,1}$ and $\underline{W}_{1,2}$ are close. This includes that for large weights, eqn (30) is satisfied if the angle between $\underline{W}_{1,1}$ and $\underline{W}_{1,2}$ is small. As soon as eqn (30) is not satisfied any more, the two neurons in the first layer make a significantly different classification. In this case, the analysis of the learning behavior can be continued after making a new vector decomposition for both neurons in the first layer. In Sections 5, 7, and 9, making a new decomposition will be explained in more detail.

It has been shown that during initial training, the hyperplanes corresponding to the neurons in the first layer attract to the same position in input space. When including the network nonlinearities, the so-formed cluster of hyperplanes may break. Two mechanism that result in breaking of the clustered hyperplanes will be analyzed. Firstly, the breaking can be caused by rotation of the hyperplanes with respect to one another. Secondly, the hyperplanes can be translated with respect to another. Thirdly, a composite mechanism of the previous two mechanisms may occur; this will not be analyzed in this paper.

The rotation-based breaking can be monitored by the angle $\varphi$ between hyperplanes in a cluster. An analysis of rotation-based breaking of clustered hyperplanes and a discussion of the relation with the encounter of temporary minima follows in Section 5. Section 6 presents illustrative examples of rotation-based breaking of a cluster of hyperplanes.

Translation-based breaking assumes that the hyperplanes move away from each other in a parallel way, and can be monitored by the distance between the hyperplanes. The analysis and brief discussion of the translation-based breaking mechanism is presented in Section 7 and an example is presented in Section 8.

\section{ROTATION-BASED BREAKING}

In this section, the mechanism behind rotation-based breaking of a cluster of hyperplanes will be analyzed. Because $\beta_{1}^{h}$ corresponds to the norm of the weight vec- tor component perpendicular to the attractor hyperplane and $\left|\underline{\Lambda} \underline{W}^{\varepsilon}\right|$ is related to the norm of the weight vector component in parallel to the attractor hyperplane, with eqns (27a) and (27b), the angle between the two hyperplanes of the two neurons in the first layer is

$$
\varphi=2 \cdot \operatorname{atan}\left(\frac{\left|\underline{\Lambda} \underline{W}^{c}\right|}{2 \beta_{1}^{h}}\right) .
$$

With the back-propagation learning algorithm, the weight vector increments during training are small. Henceforth, for the increment of the angle between the two hyperplanes for one adaptation step:

$$
\Delta \varphi=\frac{\partial \varphi}{\partial\left|\underline{\underline{\Lambda}} \underline{W}^{\prime}\right|} \cdot \Delta\left|\underline{\Lambda} \underline{W}^{e}\right|+\frac{\partial \varphi}{\partial \beta_{1}^{h}} \cdot \Delta \beta_{1}^{h} .
$$

The partial derivatives on the right-hand side of eqn (33) can directly be derived from eqn (32):

$$
\begin{aligned}
\frac{\partial \varphi}{\partial\left|\underline{\Lambda} W^{c}\right|} & =\frac{4 \beta_{1}^{h}}{4 \beta_{1}^{h^{2}}+\left|\Lambda W^{c}\right|^{2}}, \\
\frac{\partial \varphi}{\partial \beta_{1}^{h}} & =\frac{-4\left|\underline{\underline{\Lambda}} \underline{W}^{c}\right|}{4 \beta_{1}^{h^{2}}+\left|\underline{\Lambda} W^{c}\right|^{2}} .
\end{aligned}
$$

The two increments $\Delta\left|\underline{\Lambda} \underline{W}^{c}\right|$ and $\Delta \beta_{1}^{h}$ have to be derived from the back-propagation learning algorithm. With eqns (20) and (31), for the increments of $\underline{W}^{c}$, during learning of one training example,

$$
\begin{aligned}
\Delta W_{1, j}^{\text {c.p }}= & \eta(D-Y) f^{\prime}\left(\alpha_{2,1}^{h} \cdot \beta_{2,1}^{h}+\alpha_{2,1}^{\text {bias }} \beta_{2,1}^{\text {bias }}\right) \frac{\beta_{2,1}^{h}}{\sqrt{2}} \\
& \cdot f^{\prime}\left(\alpha_{1}^{h} \cdot \beta_{1}^{h}+\alpha_{1}^{\text {bias }} \beta_{1}^{\text {bias }}+\underline{W}_{\mathrm{i}, j}^{\boldsymbol{c}} \cdot \underline{U}_{1}^{F}\right) \underline{U}_{1}^{F}
\end{aligned}
$$

where $f^{\prime}(\cdot)$ is the first derivative of the output of the transfer function with respect to its input. The increment of $\left|\underline{\Lambda} \underline{W}^{c}\right|$ for one training example, $\Delta\left|\underline{\Lambda} \underline{W}^{c}\right|^{p}$, can be obtained from eqn (25):

$$
\Delta|\underline{\Lambda} W|^{p}=\Delta\left|\underline{W}_{i, 1}^{c, p}-\underline{W_{i, 2}^{k}, 2}\right| .
$$

A first-order approximation of eqn (37) is

$$
\begin{array}{r}
\Delta\left|\underline{\Lambda} \underline{W}^{c}\right|^{p}=\eta(D-Y) f^{\prime}\left(\alpha_{2,1}^{h} \cdot \beta_{2,1}^{h}+\alpha_{2,1}^{\text {bias }} \beta_{2,1}^{\text {bias }}\right) \frac{\beta_{2,1}^{h}}{\sqrt{2}} \\
\cdot f^{\prime \prime}\left(\alpha_{1}^{h} \cdot \beta_{1}^{h}+\alpha_{1}^{\text {bias }} \beta_{1}^{\text {bias }}\right)\left(\underline{U}_{1}^{F} \cdot \underline{\Lambda} \underline{W}^{c}\right)\left|\underline{U}_{1}^{F}\right|,
\end{array}
$$

where $f^{\prime \prime}(\cdot)$ denotes the second derivative of the output of the transfer functions of the neurons with respect to the input. This first-order approximation is sufficiently accurate as long as the angle between the hyperplanes is small.

The increment of the difference vector $\Lambda W^{\varepsilon}$ can be calculated by summation of eqn (38) over all training examples. For reasons of mathematical notation and operation, we use the integral equivalent of the sum

$$
\Delta\left|\underline{\Lambda} \underline{W}^{e}\right|=\int_{p} \Delta\left|\underline{\Lambda} \underline{W}^{e}\right|^{p} P(p) d p .
$$

In eqn (39), $P(p)$ denotes the probability density func- 
tion of the $p$ th training example. Substitution of eqn $(38)$ in eqn (39) results in

$$
\begin{aligned}
\Delta\left|\underline{\Lambda} \underline{W}^{c}\right|=\int_{\alpha\}} C \underline{\underline{w} \underline{\varepsilon}}\left(\alpha_{1}^{h}\right) \int_{U_{1}^{F}}\left|U_{1}^{F}\right|\left(\underline{U}_{1}^{F} \cdot \underline{\Lambda} \underline{W}^{c}\right) \\
\\
\times P\left(\alpha_{1}^{h}, \underline{U}_{1}^{F}\right) d \underline{U}_{1}^{F} d \alpha_{1}^{h}
\end{aligned}
$$

with

$$
\begin{aligned}
C_{\underline{\underline{E}}}\left(\alpha_{1}^{h}\right)=\eta(D-Y) f^{\prime}\left(\alpha_{2,1}^{h} \cdot \beta_{2,1}^{h}+\alpha_{2,1}^{\text {bias }} \beta_{2,1}^{\text {bias }}\right) & \\
& \times \frac{\beta_{2,1}^{h}}{\sqrt{2}} f^{\prime \prime}\left(\alpha_{1}^{h} \cdot \beta_{1}^{h}+\alpha_{1}^{\text {bias }} \beta_{1}^{\text {bias }}\right) .
\end{aligned}
$$

Note that the desired response, or target response, $D$ in eqn (40), is different for the two classes to be classified. Similarly, the adaptation of $\beta_{1}^{h}$ of the two neurons in the first layer can be derived. The increment $\Delta \beta_{i, j}^{h, p}$ on one training example is

$$
\begin{aligned}
\Delta \beta_{1, j}^{h, p}=\eta(D-Y) f^{\prime}\left(\alpha_{2,1}^{h} \cdot \beta_{2,1}^{h}+\alpha_{2,1}^{\text {bias }} \beta_{2,1}^{\text {bias }}\right) \frac{\beta_{2,1}^{h}}{\sqrt{2}} \\
\quad \times f^{\prime}\left(\alpha_{1}^{h} \cdot \beta_{1}^{h}+\alpha_{1}^{\text {bias }} \beta_{1}^{\text {bias }}+\underline{W}_{1, j}^{c} \cdot \underline{U}_{1}^{F}\right) \alpha_{1}^{h} .
\end{aligned}
$$

The $\Delta \beta_{1}^{h}$ over the total training set can be approximated by

$$
\Delta \beta_{1}^{h} \approx \int_{\alpha\}} \int_{U_{1}^{F}} C_{\beta 1}\left(\alpha_{1}^{h}\right) P\left(\alpha_{1}^{h}, \underline{U}_{1}^{F}\right) d \underline{U}_{1}^{F} d \alpha_{1}^{h}
$$

with

$$
\begin{aligned}
C_{\beta 1}\left(\alpha_{1}^{h}\right)=\eta(D-Y) f^{\prime}\left(\alpha_{2,1}^{h} \cdot\right. & \left.\beta_{2,1}^{h}+\alpha_{2,1}^{\text {bias }} \beta_{2,1}^{\text {bias }}\right) \frac{\beta_{2,1}^{h}}{\sqrt{2}} \\
& \times f^{\prime}\left(\alpha_{1}^{h} \cdot \beta_{1}^{h}+\alpha_{1}^{\text {bias }} \beta_{1}^{\text {bias }}\right) \alpha_{1}^{h} .
\end{aligned}
$$

By substitution of eqns (34) and ( 35 ) into eqn (33), it can be derived that

$$
\Delta \varphi \approx \sin (\varphi) \cdot\left[\frac{\Delta\left|\underline{\underline{\Lambda}} \underline{W}^{e}\right|}{\left|\underline{\Delta} \underline{W}^{c}\right|}-\frac{\Delta \beta_{1}^{h}}{\beta_{1}^{h}}\right]
$$

where the adaptation of $\beta_{1}^{h}$ and $\underline{\Lambda} \underline{W}^{c}$ are given by eqns (42) and (40). In the next sections, the interpretation of the results denoted by eqn (43) are discussed. Simulations that illustrate the effect of eqn (43) on the learning behavior are presented in Section 6 .

\subsection{Discussion}

The effect of eqn (43) on the learning behavior of the two-layer neural network will now be explained. It is important to note that the normalized increments of $\left|\underline{\Lambda} \underline{W}^{c}\right|$ and of $\beta_{1}^{h}$ at the right-hand side of eqn (43) are independent of $\varphi$. Firstly, the two normalized increments on the right-hand side of eqn (43), and secondly, the dynamics of the angle $\varphi$ during training will be discussed.

\subsection{Normalized Increment of $\left|\underline{\Lambda} \underline{W}_{e}\right|$}

The first term between brackets in eqn (43) is the normalized increment of the $\Lambda \underline{W}^{e}$ vector over all training examples,

$$
\Delta\left|\underline{\Lambda} \underline{W}^{e}\right|_{\text {norm }}=\frac{\Delta\left|\underline{\underline{\Lambda}} \underline{W}^{e}\right|}{\left|\underline{\underline{\Lambda}} \underline{W}^{e}\right|} .
$$

It can be derived from eqn (38) that adaptation in response to one training example can result in either an increment or a decrement of $\left|\underline{\Lambda} \underline{W}^{c}\right|$. For the training examples for which the integrand in eqn (39) equals zero, the superscript 0 will be used. For the examples $\underline{U}_{1}^{0}$ :

$$
\Delta\left|\underline{\Lambda} \underline{W}^{c}\right|^{p}\left(\underline{U}_{1}^{0}\right)=0 .
$$

There are two trivial and one nontrivial solutions for training examples satisfying eqn (45). One trivial solution is found for examples for which the derivative of the transfer function of the neuron in the second layer is zero. Another trivial solution of eqn (45) is found for examples that are already classified correctly; for these training examples the weight adaptation is zero.

Nontrivial examples satisfying eqn (45) are those for which the first derivatives of the transfer function of the neurons in the first layer are identical [see eqns (36) and (37)]. This is true as a good approximation for all training examples for which the second derivatives of the transfer function of the neurons in the first layer equal zero [ see eqn (38)]. When using odd transfer functions in the neurons in the first layer of the network, it follows from eqns (23) and (38) that these nontrivial examples are located in the attractor hyperplane of the neurons in the first layer,

$$
f(-x)=2 f(0)-f(x) \rightarrow \underline{U}_{1}^{0} \cdot \underline{W}_{1}^{\mathrm{ATT}}=0 .
$$

Furthermore, it can be derived from eqn (38) that

$$
\begin{array}{r}
\frac{\left|D-f\left(\sqrt{2} \beta_{2,1}^{h} f\left(\alpha_{1}^{h} \beta_{1}^{h}+\alpha_{1}^{\text {bias }} \beta_{1}^{\text {bias }}\right)+\alpha_{2,1}^{\text {bias }} \beta_{2,1}^{\text {bias }}\right)\right|}{\left|D-f\left(\sqrt{2} \beta_{2,1}^{h} f(0)+\alpha_{2,1}^{\text {bias }} \beta_{2,1}^{\text {bias }}\right)\right|} \\
>1 \Leftrightarrow \Delta\left|\underline{\Lambda} \underline{W}^{\bullet}\right|^{p}>0 .
\end{array}
$$

Relation (47) denotes that training examples that lie on the "wrong" side of the attractor hyperplane, from a classification point of view, have a positive contribution to $\Delta\left|\underline{\Lambda} \underline{W}^{\mathrm{c}}\right|$. Training examples that lie on the "correct" side of the attractor hyperplane tend to reduce the norm of $\Lambda W^{z}$.

Training examples that result in weight adaptation are bounded by both the training set boundaries and by the training examples for which $(D-Y)=0$. With eqns (40), (44), and (47), it can be derived that

$$
\Delta\left|\underline{\Lambda} \underline{W}^{c}\right|_{\text {norm }}(t+\tau)>\Delta\left|\underline{\Lambda} \underline{W}^{*}\right|_{\text {norm }}(t) \quad \tau>0
$$

under condition of small initial weights (in terms of the definition in the beginning of Section 4).

\subsection{Normalized Increment of $\beta_{1}^{h}$}

The second term between brackets in eqn (43) is the normalized increment of $\beta_{1}^{h}$ over all training examples,

$$
\Delta \beta_{\mathrm{Inorm}}^{h}=\frac{\Delta \beta_{1}^{h}}{\beta_{!}^{h}} .
$$


During training, eqn (49) will be positive as long as eqn (30) is satisfied, assuming positive weights associated with neurons in the first layer for the neuron in the second layer. Using small initial weights, $\Delta \beta_{1}^{h}$ decreases during training for several reasons. Firstly, the number of correctly classified training examples increases during training, which results in an average decrease of the mean adaptation. Secondly, the mean adaptation on not yet correctly classified training examples decreases. For the normalized increment,

$$
\Delta \beta_{1 \text { norm }}^{h}(t)>\Delta \beta_{1 \text { norm }}^{h}(t+\tau) \geq 0 \quad \tau>0 .
$$

\subsection{The Angle $\varphi$ During Training}

Under assumption of small initial weights, three significantly different cases are distinguished during training. These cases are determined by the sign of the sum of the normalized increments of $\beta_{1}^{h}$ and $\left|\underline{\Lambda} \underline{W}^{\mathfrak{l}}\right|$.

1. The first situation occurs if the expression between brackets in eqn (43) is positive at the beginning of the training,

$$
\left[\frac{\Delta\left|\underline{\Delta} \underline{W}^{\imath}\right|}{\left|\underline{\Delta} \underline{W}^{c}\right|}-\frac{\Delta \beta_{1}^{h}}{\beta_{1}^{h}}\right]>0 \quad t \geq 0 .
$$

In this case, the sum will remain positive because of eqns (48) and (50). With eqns (48) and (50), it follows from eqn (47) that the situation reflected by eqn (51) only occurs if the classification made by the network for small $\varphi$ is very poor.

From eqn (43), it follows that there exists a set of $\varphi$ for which $\varphi$ is constant. These angles will be denoted as invariant angles. The invariant angles are given by

$$
\varphi=n \pi \quad n \in\{0,1,2, \ldots\} .
$$

With eqns (43), (51), and (52), it follows that angles with even $n$ are unstable and that angles with odd $n$ are stable. According to eqn (43), for any initial angle that is not an even multiple of $\pi, \varphi$ goes asymptotically to the nearest odd multiple of $\pi$. Note that if the two-layer neural net is in an equilibrium, the two hyperplanes of the neurons in the first layer coincide:

$\left\{\begin{array}{lll}\varphi=2 n \pi & n \in\{0,1,2, \ldots\} \Rightarrow & \text { unstable } \\ \varphi=(2 n+1) \pi & n \in\{0,1,2, \ldots\} \Rightarrow & \text { stable }\end{array}\right.$.

2. In the second possible situation for the training of the two-layer neural network, the square brackets term in eqn (43) is negative throughout the training,

$$
\left[\frac{\Delta\left|\underline{\Delta} \underline{W}^{e}\right|}{\left|\underline{\Delta} \underline{W}^{e}\right|}-\frac{\Delta \beta_{1}^{h}}{\beta_{1}^{h}}\right]<0 \quad t \geq 0 .
$$

With eqns (48) and (50), it follows from eqn (47) that the classification made by the neural network is good. From eqn (43) it follows that in this case the invariant angles between the two hyperplanes are also given by eqn (53). However, the stable and unstable angles are interchanged with respect to eqn (53),

$\left\{\begin{array}{lll}\varphi=2 n \pi & n \in\{0,1,2, \ldots\} \Rightarrow & \text { stable } \\ \varphi=(2 n+1) \pi & n \in\{0,1,2, \ldots\} \Rightarrow & \text { unstable }\end{array}\right.$.

In this situation, the angle between the two hyperplanes decreases asymptotically to an even multiple of $\pi$ during the entire training; the weight vector attractors of the neurons in the first layer coincide. Because the two hyperplanes of the neurons in the first layer tend to coincide during training, the classification of the training set will be carried out using redundant neurons in the first layer of the neural network; that is, the two neurons make almost identical classifications.

3. The last possible learning behavior is the most interesting with respect to the derivation of several properties concerning the learning. In this last case, the term between brackets in eqn (43) is negative in the beginning of the training, and becomes positive during training:

$$
\begin{cases}{\left[\frac{\Delta\left|\underline{\underline{\Lambda}} \underline{W}^{c}\right|}{\left|\underline{\underline{\Lambda}} \underline{W}^{c}\right|}-\frac{\Delta \beta_{1}^{h}}{\beta_{1}^{h}}\right] \leq 0} & t \leq \tau_{c r i t} \\ {\left[\frac{\Delta\left|\underline{\underline{\Lambda}} \underline{W}^{c}\right|}{\left|\underline{\Lambda} \underline{W}^{c}\right|}-\frac{\Delta \beta_{1}^{h}}{\beta_{1}^{h}}\right]>0} & t>\tau_{c r i t}\end{cases}
$$

It is clear that in this case, the learning behavior sequentially surpasses the two previously mentioned cases. In the beginning of the training, the angle between the hyperplanes converges towards an even multiple of $\pi$. After a certain point in the training, however, the angle between the hyperplanes is driven to an odd multiple of $\pi$. This means that the weight vector attractors of the neurons in the first layer coincide for $t \leq \tau_{\text {crit }}$ and are noncoinciding elsewhere.

\subsection{Discussion of Negative Elements in $\underline{B}_{2,1}^{k}$}

In the analysis, all elements of $\underline{B}_{2,1}^{h}$ were assumed to be positive. In general, however, the elements of $B_{2,1}^{h}$ can be either positive or negative. The effect of negative weights associated with neurons in the first layer on the learning behavior of two-layer neural networks is small; a negative element in $\underline{B}_{2,1}^{h}$ means that for the associated neuron in the first layer the weight vector points in the opposite direction compared to a positive weight:

$$
W_{2,1}[j] \cdot \beta_{1, j}^{h}>0 \text {. }
$$

Hence, for neurons in the first layer connected to a negative weight, both $\beta_{i, j}^{h}$ and $W_{i, j}^{c}$ have opposite signs compared to neurons for which outputs are connected to positive weights. The effect on the preceding analysis is that the stable and unstable equilibria of the hyperplanes in eqns (53) and (55) are interchanged for every nonpositive elements in $B_{2,1}^{h}$. Note that also the expression for $\Lambda W^{e}$ in eqn (26) must be changed and that the signs of $\beta_{1, j}^{h}$ must be compensated for. 
Another effect, not analyzed in this article, is that the training time needed to obtain a certain level of performance for mixed positive and negative elements in $B_{2,1}^{h}$ may differ from the training time for the case of only positive elements.

\subsection{Temporary Minima}

In this section, it is shown that the learning behavior depicted in eqn (56) leads towards the encounter of a temporary minimum. To happen upon the learning behavior of eqn (56), the training set must satisfy a number of conditions. Firstly, it follows from eqns (47), (48), (50), and (56) that the training set needs to be nonlinearly separable. Secondly, the performance of the neural network with clustered hyperplanes must be nonoptimum to break the cluster of hyperplanes. Thirdly, the performance of the neural network with clustered hyperplanes in the first layer must be good enough to ensure that the situation depicted in eqn (56) is encountered; a very poor classification of the training set using clustered hyperplanes results in eqn (51).

While training a network for $\tau \leq \tau_{c r i t}$ in eqn (56), the angle between the hyperplanes corresponding to the neurons in the first layer decreases monotonically. This means that the network builds up a redundancy of the neurons in the first layer. The classification of the training set made by the network approaches, in this phase, the classification made by a network with redundancy. The analysis as presented so far requires that eqn (30) be satisfied, which is true for either small weights or a small angle between the weight vectors; this latter includes a small angle $\varphi$ between the hyperplanes. It can easily be shown that eqn ( 30 ) is satisfied for $\tau \leq \tau_{\text {cri }}$ in eqn (56) assuming small initial weights.

In the subsequent phase, $t>\tau_{\text {crit }}$ in eqn (56), the angle $\varphi$ increases monotonically, which indicates gradual abolishing of the redundancy in the first layer. The analysis as presented in this article remains valid as long as the angle $\varphi$ remains small [see eqn (30)]. After eqn (30) is no longer satisfied, the hyperplanes corresponding to the neurons in the first layer make a significantly different classification; the input space has been subdivided, or partitioned (Liang, 1991), into two parts, each classified by one neuron in the first layer. In this case, the analysis of the learning behavior of a two-layer neural net can be continued by making new vector decompositions for the two neurons in the first layer; the directions of the $B_{1, j}^{h}$ component vectors are then related to the part to be classified by the corresponding neuron and is hence different for both neurons in the first layer.

Due to abolition of the redundancy of the neurons in the first layer, the classification of the training examples can be improved significantly. This vanishing of redundancy results in escaping from the temporary minima (Murray, 1991a). It can directly be seen from the term $\sin (\varphi)$ in eqn (43) that abolishing the redundancy, that is, increasing of the angle $\varphi$, is generally a slow process. This explains why neural nets may stick in a temporary minimum during training for a relatively long time.

\section{ROTATION-BASED BASED BREAKING: AN ILLUSTRATIVE EXAMPLE}

In this section, the equations describing the dynamics of the two-layer neural network during training, eqns (40), (42), and (43), will be used to describe the learning behavior for a simple classification problem that results in subsequently clustering and rotation-based breaking of the formed cluster of hyperplanes.

\subsection{Training Set to be Learned}

To demonstrate the conclusions of Sections 4 and 5 , we consider the learning behavior of a two-layer feedforward neural net for classification of two classes in two-dimensional space. The classes in this training set $A$ are indicated in Figure 3 by the two shaded areas. Note that all neurons in the neural network have a three-dimensional input and weight vector. The input vectors for the neurons in the first layer have two external input elements and one bias input element [see eqn (1)]. The neuron in the second layer has two inputs from the neurons in the first layer and one bias input [see eqn (2)].

\subsection{Initialization}

Before starting the training of the neural network, the weights of the neurons in the network have to be initialized. It is assumed that the initial weights are small enough to allow for linearization of the neural network at the beginning of training. According to Section 4, under this condition, the weight vectors of the neurons in the first layer adapt almost identically in the beginning of training. Furthermore, the elements in $\underline{W}_{2,1}$, which are not related to the bias input, are also adapting almost identically in this phase.

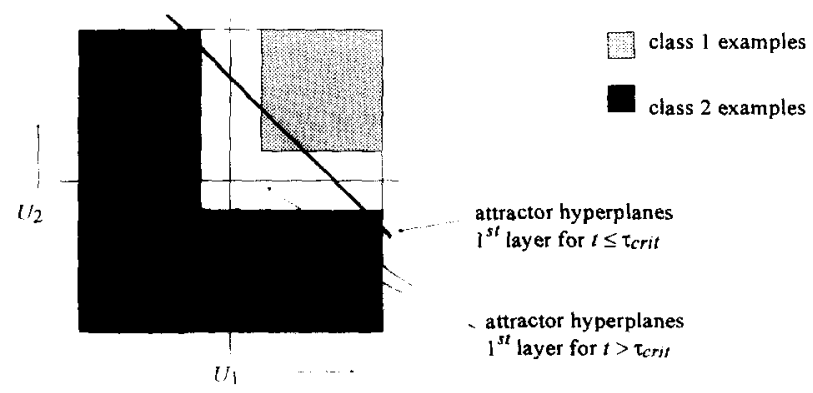

FIGURE 3. Training set $A$ and the corresponding attractor hyperplanes of the neurons in the first layer; thick line for $t \leq \tau_{\mathrm{crtt}}$ and thin lines for $t>\tau_{\mathrm{crth}}$. 


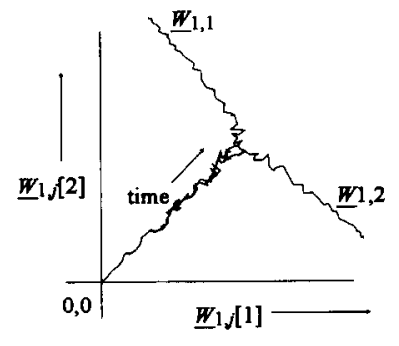

(a)

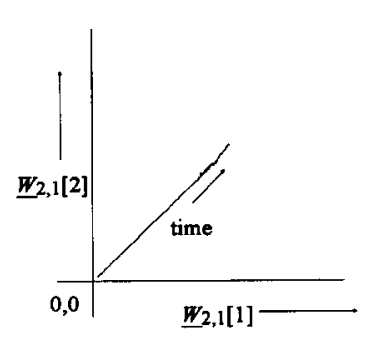

(b)
FIGURE 4. Simulated dynamics of the (nonbias) weights of the neurons in weight space.

To illustrate this, Figure 4 presents the simulated dynamics of the weights (excluding the bias-related weights) in weight space. These simulation results are obtained using a neural network simulation program that trained the specified neural net on the training set of Figure 3.

Figure 4a clearly illustrates that in the beginning of training using very small initial weights, the nonbiasrelated vector component in $\underline{W}_{1, j}$, that is, the vectors $\underline{W}_{1, j}^{r}$, adapt identically. After a certain training time, the so-formed cluster of hyperplanes abruptly breaks into two hyperplanes, which make a significantly different classification. The direction in which $\underline{W}_{1, j}^{r}$ adapts in weight space is dependent on the training set for both the phase of training in which hyperplanes are clustered and for the phase of training in which the neurons in the first layer make a significantly different classification.

Figure $4 \mathrm{~b}$ shows that the nonbias-related weights in $W_{2,1}$, that is, the elements of $\underline{W}_{2,1}^{r}$, adapt identically during the training. In the beginning of training, this is due to the fact that very small initial weight are used, which allows for linearization. After breaking of the cluster of hyperplanes, the nonbias-related weights in $W_{2,1}$ continue to adapt identically, which is due to symmetry reasons (i.e., the fact that for the used training set the two parts that are classified by the nonclustered hyperplanes are equally important for the classification of the total training set).

\subsection{Phase Portrait}

Under the condition of small initial weights, the learning behavior of the two-layer neural network is deterministic according to eqns (40), (42), and (43). Note that the order of presentation of the training examples is not important; the network adapts according to eqn (6) in which the weight adaptation is gradient descent for the squared error over all training examples.

Because of the deterministic behavior of the learning having global learning and negligibly small initial weights, the $\beta_{k, j}^{h}, \beta_{k, j}^{\text {bias }}$, and $\left|\underline{W}_{k, j}^{e}\right|$ of all neurons in the neural network are strongly correlated. The state of the neural network, which is a function of the $\beta_{k, j}^{h}, \beta_{k, j}^{\text {bias }}$, and $\left|\underline{W}_{k, j}^{c}\right|$ of all neurons, can therefore be expressed in, for example, $\beta_{1}^{h}$ and $\varphi$. By doing so, a two-dimensional phase portrait (Guckenheimer \& Holmes, 1983) of the learning behavior of the two-layer neural network on the training set presented in Figure 3 can be constructed; this phase portrait is given in Figure 5. Because eqns (40), (42), and (43) have been derived under condition of eqn ( 30 ), this phase portrait is valid only after the linearization phase (see Section 4) and until the hyperplanes make a significantly different classification. For subsequent phases of training, a new vector decomposition is to be made for every cluster of redundant neurons and hence new phase portraits result.

In Figure 5, the phase trajectories start for values of $\beta_{1}^{h}$ that marginally do not allow linearization of the network, a situation that corresponds to the begin situation for clustering and breaking behavior according to eqn (43). In the figure, the initial angles $\varphi$ have been chosen in a large range for reasons of illustration.

During training, $\beta_{1}^{h}$ moves asymptotically towards $\beta_{\max }$, which corresponds to the value of $\beta_{1}^{h}$ obtained for a redundant neural network after an infinite training. For nonredundant networks, the angle $\varphi$ moves towards an even integer multiple of $\pi$ for $\beta_{1}^{h}<\beta_{\text {crit }}$, with $\beta_{\text {crit }}$ corresponding to $\tau_{\text {crit }}$ in eqn (56). After $\beta_{\text {crit }}$ has been reached, $\varphi$ moves towards an odd multiple of $\pi$.

The phase portrait is valid as long as (30) is satisfied. The validity borders in fig. 5 mark the borders below which condition (30) is satisfied. As soon as a phase trajectory crosses one of these validity borders, a new vector decomposition has to be made.

\subsection{Phase Portrait Assuming Major Simplifications}

The phase portrait in Figure 5 has been constructed by using the equations in Sections 4 and 5 , which is very time consuming. However, with some major simplifications, it is possible to very simply construct an approximating phase portrait: assume that $\beta_{1}^{h}$ approaches $\beta_{\max }$ exponentially and assume that the normalized increment of the norm of the $\Lambda \underline{W}^{e}$ vector is a

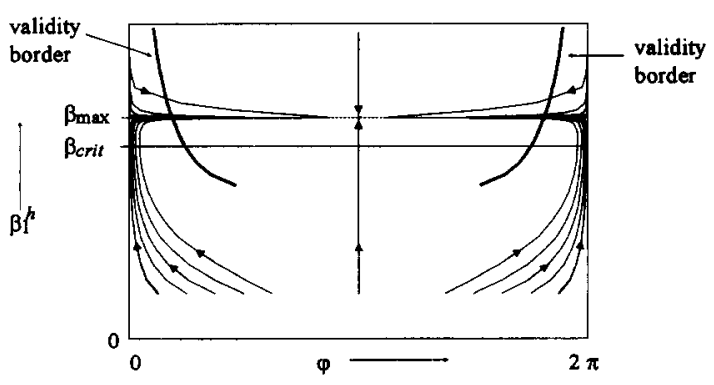

FIGURE 5. Phase portrait for the learning behavior of a twolayer neural network: rotation-based breaking of clustered hyperplanes. 
linearly increasing function of $\beta_{1}^{h}$. In this way the phase portrait can be constructed using

$$
\left\{\begin{array}{l}
\Delta \varphi=A \sin (\varphi)\left[\beta_{1}^{h}-\beta_{c r i t}-\frac{\beta_{\max }-\beta_{1}^{h}}{\beta_{1}^{h}}+\frac{\beta_{\max }-\beta_{c r i t}}{\beta c r i t}\right] \\
\Delta \beta_{1}^{h}=\beta_{\max }-\beta_{1}^{h}
\end{array}\right.
$$

(with $A$ a scalar scale value), which generally gives an acceptably accurate approximation of the actual phase portrait for training sets that result in rotation-based breaking of clusters of hyperplanes. With this set of difference equations, among others, the changing of the distribution of the examples from which the network learns and the changing adaptation on these training examples are simplified significantly. However, one can easily (and fast) get a good impression of the neural net's learning behavior using this set of difference equations.

\subsection{Learning Behavior for $0<\beta_{\text {crit }}<\beta_{\max }$}

It follows from eqn (47) that in the case of the nonlinearly separable training set $\mathrm{A}, \beta_{\text {crit }}$ is smaller than $\beta_{\max }$. For sets with $\beta_{\text {crit }}<\beta_{\max }$, the angle $\varphi$ goes asymptotically to an even multiple of $\pi$ for $t \leq \tau_{\text {crit }}$ in eqn (55). During continued training, $t>\tau_{\text {crit }}, \varphi$ tends to converge to an odd multiple of $\pi$. This means that for the first layer with redundant neurons at $t=\tau_{\text {crit }}$, the redundancy is lifted for $t>\tau_{\text {crit }}$.

\subsection{Learning Behavior for $\beta_{c r i t}>\beta_{\max }>0$}

For some training sets, particularly those with linearly separable classes, $\beta_{\text {crit }}$ will be higher than $\beta_{\max }$ [ see eqn (47)]. In this case, on condition of small initial weights, the network will tend to build up redundancy in the first layer during the entire training. In Figure 6, an example of a (linearly separable) training sets for which $\beta_{\text {crit }}>\beta_{\max }$ is given.

For linearly separable classes, the inequality $\beta_{\text {crit }}>$ $\beta_{\max }$ is not a problem for the classification to be learned as any neural network with redundant neurons in the first layer can classify the separable training set correctly.

The starting conditions for the analysis, which are satisfied when using small enough initial weights, will generally not be satisfied when using a priori knowledge.

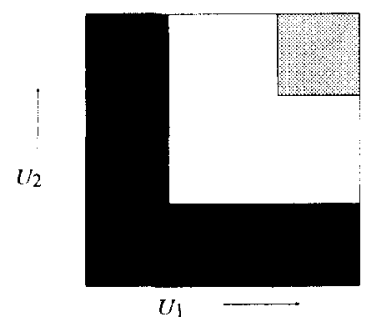

FIGURE 6. Training set $\mathrm{B}$ with $\beta_{\mathrm{cm}}>\beta_{\max }$.

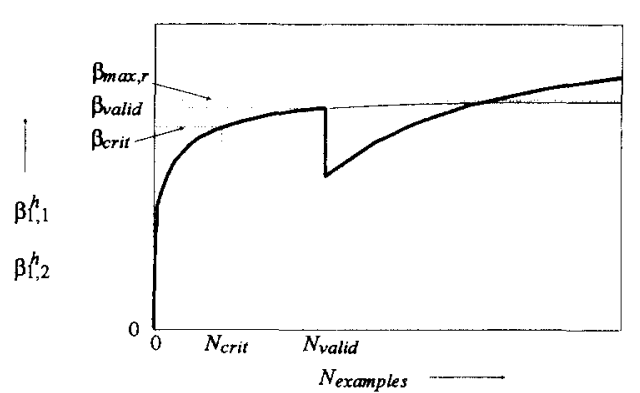

FIGURE 7. $\beta_{1, n}^{h}$ as a function of the number of training examples.

In case of proper use of a priori knowledge in initial weights, the hyperplanes corresponding to the neurons in the first layer may have different attractor hyperplanes throughout the training. In that case no redundancy will be built up in the beginning of training.

\subsection{Phase Portrait Allowing Negative Elements in $\underline{B}_{2,1}^{h}$}

As discussed in Section 4, the stable and unstable invariant angles in eqns (53) and (55) may interchange for negative elements in $\underline{B}_{2,1}^{h}$. Directly after initializing the neural network using negligibly small weights for all neurons in the neural network, $\underline{B}_{2,1}^{h}$ cannot be defined.

The direction in which $\underline{B}_{2,1}^{h}$ points after a short training period is now determined by two competing weight vector attractors. One attractor attracts to a state for which the stable angles are at even multiplies of $\pi$ [see eqn (53)], corresponding to an even number of negative weights in $\underline{W}_{2,1}$. The other attractor attracts to equilibrium states for which odd multiplies of $\pi$ are stable; this corresponds to an odd number of negative weights in $W_{2,1}$.

Therefore, as soon as the weight vectors in the network are attracted to a stable angle, the resulting phase portrait is either the phase portrait shown in Figure 5 or a phase portrait identical to the one in Figure 5 but shifted in the $\varphi$ direction over $\pi$ due to interchanging of the stable and unstable angles.

\subsection{Required Training Time}

The training time required to obtain a certain level of performance for a particular training set has been calculated with the help of eqns (42) and (43) using a special purpose computer program. For training set $A$, the values of $\beta_{1, n}^{h}$ for the neurons in the first layer have been calculated as a function of the number of training examples. In Figure 7, these (coinciding) curves have been plotted (thick lines).

In Figure $7, \beta_{\text {crit }}$ corresponds to the value of $\beta_{1}^{h}$ at $\tau_{\text {crit }}$ in eqn (56) and $\beta_{\text {valid }}$ corresponds to the value of $\beta_{1}^{h}$ for which eqn (30) is marginally satisfied (see also Figure 5). As soon as $\beta_{1}^{h}$ equals $\beta_{\text {valid }}$, the two neurons 
in the first layer start to make a significantly different classification. At this point in training, a new vector decomposition of the weight vectors of the neurons in the first layer has to be made to be able to continue the analysis. This new vector decomposition causes the drop of the $\beta_{1, n}^{h}$ curves that is by a factor $\cos (\theta)$, where $\theta$ is the rotation of the $B_{1, n}^{h}$ vectors at $\beta_{\text {valid }}$; for this particular example $\theta=45^{\circ}$.

After making a significantly different classification of the two hyperplanes, the values of $\beta_{1, n}^{h}$ continue to increase to generally larger values than $\beta_{\max , r}$. The coincidence of $\beta_{1,1}^{h}$ and $\beta_{1,2}^{h}$ after the breaking of the cluster is due to the symmetry of the particular training set.

\subsection{The Mean Squared Error}

An indication for the performance of a feedforward neural network is the MSE of the output signal of the network calculated over all training examples (Rumelhart et al., 1986; Hirose et al., 1991; Murray, 1991; Sheu, 1991).

For the specified network and training set $\mathrm{A}$, the MSE has both been calculated and simulated as a function of the number of training examples. In Figure 8, the calculated and simulated MSE versus training time curves and the angle between the hyperplanes $\varphi$ are plotted; note the logarithmic $y$-axis for $\varphi$.

For a network with redundant neurons in the first layer, it is not possible to classify all training examples correctly. Therefore, the MSE of network with redundant neurons does not reach zero but will stick in a temporary minimum. Assuming proper initialization, the network will however lift the redundancy during training.

Assuming proper initialization, the MSE versus training time curve in the beginning of the training is approximately identical to the curve obtained for a network with redundant neurons in the first layer because the network builds up redundancy. The MSE will therefore approach the MSE corresponding to a network with redundant neurons in the first layer. Dur-

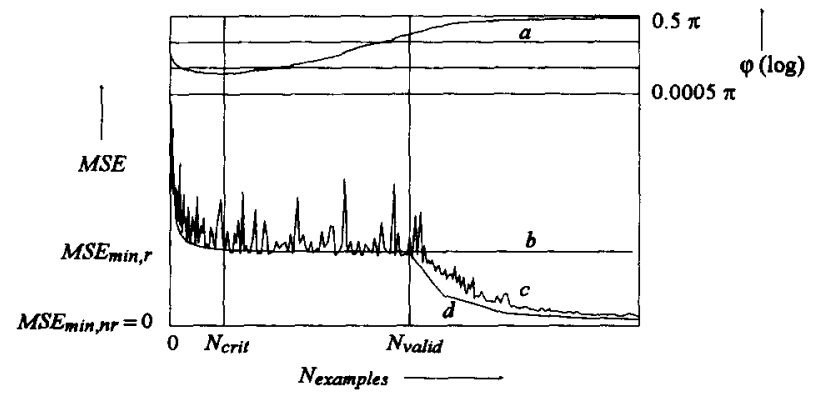

FIGUAE 8. The angle $\varphi$ (a), the calculated MSE for a redundant network (b), and the simulated (c) and calculated (d) MSE for a nonredundant network as a function of the number of training examples. ing training, the redundancy will slowly be abolished as soon as $\beta_{1}^{h} \approx \beta_{\text {crit }}$, marked with $N_{\text {crit }}$ in Figure 8 . This slow annihilation of the redundancy can be noticed in the MSE versus training time curve as a temporary minimum (Figure 8).

The analysis as presented in this section is valid until the angle $\varphi$ is so large that eqn (30) is no longer satisfied; in Figure 8, this point is marked with $N_{\text {valid }}$. After $N_{\text {valid }}$ has been reached, the hyperplanes corresponding to the two neurons in the first layer make a significantly different classification; the input space has been subdivided, or partitioned (Liang, 1991) over the two neurons in the first layer. The analysis of the learning behavior can now be continued after making a new vector decomposition with the $\underline{B}_{1, j}^{h}$ vectors related to the (two) attractor hyperplanes corresponding to the two parts of the subdivided input space.

Due to the removal of the redundancy of neurons in the first layer of the network, the classification of the training examples can be improved considerably. The MSE therefore decreases relatively quickly after $N_{\text {valid }}$. Note that because training set $A$ can be classified with a two-layer neural net having two neurons in the first layer, the MSE will approach zero after $N_{\text {valid }}$.

In the temporary minimum, it might be concluded from the MSE versus training time curve that the increase of the performance of the total network approaches zero. However, as can be derived from curve a in Figure 8 and as shown by the analyses in this paper, a fundamental performance improving process takes place in the interval between $N_{\text {crit }}$ and $N_{\text {valid. }}$. This is observed in the adaptation of the angle $\varphi$, although it is not noticeable in the MSE versus training time curve.

From eqns (47), (50), and (56), it can be derived that the better the classification by a network with redundant neurons, the lower $M S E_{\min }$ and the higher $N_{\text {crit }}$ will be. Therefore, for relatively good classification of a nonlinearly separable training set by a network with redundant neurons, the network sticks in a temporary minimum for a long (training) time. For training sets for which the classification made using a reduced network is poor, the network sticks in a temporary minimum only during a relatively short training time. In the MSE versus training time curve, this sticking phenomenon may not even be recognized.

\section{TRANSLATION-BASED BREAKING}

For some training sets, the declustering of the hyperplanes is not rotation based, as analyzed in Section V, but in a parallel manner, called translation-based breaking. In this section, the mechanism behind translation-based breaking of clustered hyperplanes is analyzed. The analysis again assumes very small initial weights (as defined in Section 4) and follows the same line as the analysis of the rotation-based breaking of clustered hyperplanes in Section 5. 
For the translation-based breaking of clustered hyperplanes, it will be assumed that the clustered hyperplanes move away from each other in a parallel manner. In this way, the norms of the $\underline{W}^{e}$ vector components are assumed to be zero. The distance between the hyperplanes is now determined by the $\beta_{1, j}^{h}$ and by the $\beta_{1, j}^{\text {bias }}$ of the two neurons with clustered hyperplanes. It is straightforward to derive that (under condition of a zero norm for the $\underline{W}^{e}$ vector component) the distance between a hyperplane and the origin of input space is given by

$$
d\left(\underline{Q}, \text { hyperplane }_{1, j}\right)=\left|\frac{\beta_{1, j}^{\text {bias }}}{\beta_{1, j}^{h}}\right| .
$$

With eqn (26), the distance between the hyperplanes of the two neurons in the first layer (in parallel with respect to each other), is then

$$
\operatorname{dist}=\left|\frac{\Lambda \beta_{1}^{\text {bias }}}{\beta_{1}^{h}}\right| .
$$

During training, the weight vectors of all neurons in the neural network adapt. As a direct result, the distance between the two hyperplanes in parallel also adapts during training. Analogous to the derivation in Section 5 , the adaptation of the distance dist is:

$$
\Delta \text { dist } \approx \operatorname{dist} \cdot\left[\frac{\Delta \Lambda \beta_{1}^{\text {bias }}}{\Lambda \beta_{1}^{\text {bias }}}-\frac{\Delta \beta_{1}^{h}}{\beta_{1}^{h}}\right] .
$$

Equations (41) and (42) describe the adaptation of $\beta_{1}^{h}$. The adaptation of $\Lambda \beta_{1}^{\text {bias }}$ on the $p^{\text {th }}$ training example is then analogous to eqn (38) to (43) given by:

$$
\begin{aligned}
\Delta \Lambda \beta_{1}^{\text {bias }, p} & =\eta(D-Y) f^{\prime}\left(\alpha_{2,1}^{h} \beta_{2,1}^{h}+\alpha_{2,1}^{\text {bias }} \beta_{2,1}^{\text {bias }}\right) \frac{\beta_{2,1}^{h}}{\sqrt{2}} \\
& \times f^{\prime \prime}\left(\alpha_{1}^{h} \beta_{1}^{h}+\alpha_{1}^{\text {bias }} \beta_{1}^{\text {bias }}\right)\left(\alpha_{1}^{\text {bias }} \Lambda \beta_{1}^{\text {bias }}\right) \alpha_{1}^{\text {bias }} .
\end{aligned}
$$

The average adaptation $\Lambda \beta_{1}^{\text {bias }}$ is then

$$
\Delta \Lambda \beta_{1}^{\text {bias }}=\int_{p} \Delta \Lambda \beta_{1}^{\text {bias,p }} P(p) d p .
$$

\subsection{Discussion}

The effect of eqn (59) on the learning behavior is comparable to the effect of eqn (43). Similarly to the discussion in Section 5, three possible situations can occur during training.

1. Firstly, the term between brackets in eqn (59) is positive throughout the training. In this case, the hyperplanes diverge (in parallel with respect to each other) at any point in training. In this case, the classification of the training set with clustered hyperplanes of neurons in the first layer is very poor. The stable and unstable invariant distances are then given by:

$$
\begin{cases}\text { dist }=0 & \Rightarrow \text { unstable } \\ \text { dist } \rightarrow \infty & \Rightarrow \text { stable }\end{cases}
$$

Note that as soon as the distance dist between the two hyperplanes is so large that (30) is no longer satisfied, a new vector decomposition is to be made for both neurons in the first layer.

2. Secondly, the term between brackets in eqn (59) is negative during training. It may be evident that the hyperplanes of the two neurons in the first layer contract towards the same attractor throughout training. In this case, the classification of the training set with one cluster of hyperplanes (virtually one neuron in the first layer) is good. Similar to eqns (53) and (55), the stable and unstable invariant distances are interchanged with respect to eqn (62).

3. Thirdly, the term between brackets in eqn (59) may be negative in only the beginning of training and becomes positive at a certain point in training. This situation is comparable to the situation depicted by eqn (56) in Section 5:

$$
\left\{\begin{array}{ll}
{\left[\frac{\Delta \Lambda \beta_{1}^{\text {bias }}}{\Lambda \beta_{1}^{\text {bias }}}-\frac{\Delta \beta_{1}^{h}}{\beta_{1}^{h}}\right] \leq 0} & t \leq \tau_{\text {crit }} \\
{\left[\frac{\Delta \Lambda \beta_{l}^{\text {bias }}}{\Lambda \beta_{1}^{\text {bias }}}-\frac{\Delta \beta_{1}^{h}}{\beta_{1}^{h}}\right]>0} & t>\tau_{\text {crit }}
\end{array} .\right.
$$

In this case, a temporary minimum is encountered during training in a way similar to the way described for rotation-based breaking of clustered hyperplanes in Section 5.

\subsection{Discussion of Negative Weights in $B_{2,1}^{h}$}

It was assumed for the analysis in this section that the elements in $B_{2,1}^{h}$ are positive. For negative elements in this unit vector, the hyperplanes of associated neurons in the first layer are rotated $180^{\circ}$ with respect to a positive element. Due to the calculation of the absolute value in eqn (58), the sign of any element in $B_{2,1}^{h}$ does not have any effect for the translation-based breaking mechanism. Note, however, that allowing negative signs in $\underline{B}_{2,1}^{h}$ results in a different expression for $\Lambda \beta_{1}^{\text {bias }}$ and that also compensations for the signs of $\beta_{1, j}^{h}$ must be included.

Another difference resulting from negative elements in $\underline{B}_{2,1}^{h}$ (not analyzed in this paper) is that the training time required to reach a certain level of performance may be changed.

\subsection{Temporary Minima}

It has been shown in Section 5 that the learning behavior depicted by eqns (43) and (56) leads towards the encounter of a temporary minimum, which was due to subsequently building up and removing redundancy in the first layer of a two-layer feedforward neural network.

The learning behavior depicted in eqns (59) and (63) is strongly related to the behavior of eqns (43) and (56); only minor differences exist. To avoid major 
redundancy in the text of this article, we do not repeat an extensive discussion of the occurrence of temporary minima for translation-based breaking of clustered hyperplanes.

\section{TRANSLATION-BASED BREAKING: AN ILLUSTRATIVE EXAMPLE}

In this section, simulation results that illustrate the translation-based breaking of clustered hyperplanes are presented. The training set used in this example is again two-dimensional and suited for classification using a two-layer neural network with two neurons in the first layer: the XOR-problem (shown in Figure 9) has been selected.

In the very beginning of training, the hyperplanes of the two neurons in the first layer form a cluster. This cluster is positioned in input space in such a way that the solution of the neural network for the training set is optimum. With the origin of input space in the lower left corner of the training set [indicated by the coordinates $(0,0)]$, the cluster is positioned outside the training set, in the lower left corner of Figure 9. During training, the hyperplanes continue to move towards each other for $t \leq \tau_{\text {crit }}$.

The clustered hyperplanes move away from each other in a parallel way for $t>\tau_{\text {crit }}$, and move towards their final position in input space. These final positions are indicated by the two thin lines in Figure 9.

\subsection{Phase Portrait}

Similar to the learning behavior with rotation-based breaking of clustered hyperplanes, a phase portrait can be constructed of the learning behavior with translationbased breaking. In the phase portrait, the distance between the hyperplanes in parallel is plotted as a function of $\beta_{1, n}^{h}$. The simulated phase portrait of the learning behavior of a two-layer neural network as specified in the beginning of Section 6 on the training set of Figure 9 is shown in Figure 10.

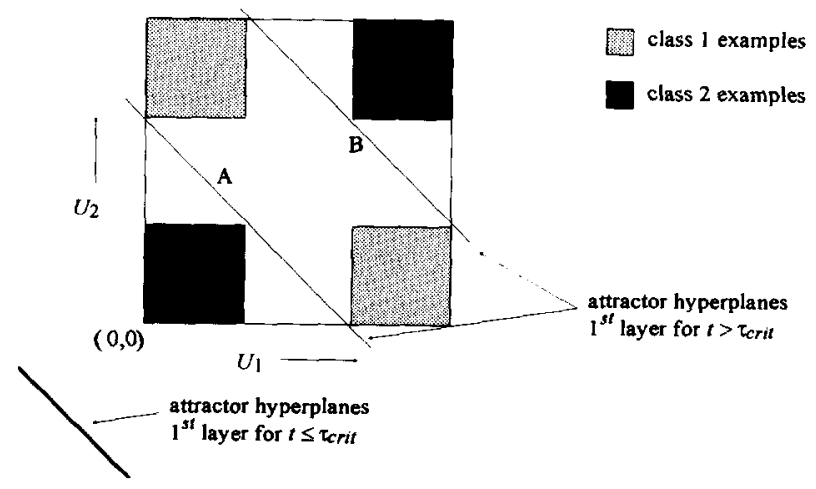

FIGURE 9. Training set C (XOR problem) and the corresponding attractor hyperplanes of the neurons in the first layer; thick line for $t \leq \tau_{\mathrm{ort}}$ and thin lines for $t>\tau_{\mathrm{cot} t}$.

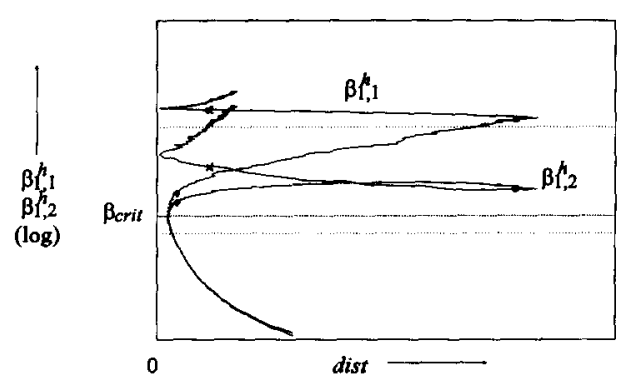

FIGURE 10. Phase portrait for the leaming behavior of a twolayer neural network: translation-based breaking of clustered hyperplanes.

In this figure, the distance dist has been plotted as a function of both $\beta_{1,1}^{h}$ and $\beta_{1,2}^{h}$ because, after breaking the cluster at $t=\tau_{\text {crit }}$, the adaptation of $\beta_{1,1}^{h}$ and $\beta_{1,2}^{h}$ is different. Note that after eqn ( 30 ) is no longer satisfied, the weight vector attractors of the two neurons in the first layer are significantly different, which results in divergence of the two trajectories in the phase portrait. To compare the trajectories after breaking of the cluster, a number of markers have been included in Figure 10: identical markers at the two trajectories correspond to specific points in training.

At a certain point in training and after declustering of the hyperplanes, the distance between the two hyperplanes decreases rapidly and then increases to its final value. This is explained as follows: directly after declustering of the hyperplanes, one of the hyperplanes moves towards attractor hyperplane $A$ in Figure 9. The other hyperplanes moves after a certain time towards attractor hyperplane B in Figure 9, for which it has to pass the hyperplanes that attracts to attractor $A$. This passing process causes the rapid decrease and increase of the dist variable in Figure 10 after declustering.

\subsection{The Mean Squared Error}

It follows from Figure 10 that the neural network sticks in a temporary minimum for relatively small $\beta_{1}^{h}$. This means that the temporary minimum is encountered in the beginning of training, just after the phase in which the network can be linearized (see Section 4). In Figure 11 , the simulated MSE of the network is plotted as a function of the number of presented training examples.

The markers that were plotted in Figure 10 are also included in the MSE versus training examples curve. Note that for this particular training set, the major part of the sticking time is required to build up the redundancy in the first layer of the two-layer neural network, whereas for the rotation-based mechanism the redundancy-lift required most sticking time.

\section{EXTENSION TOWARDS LARGER NETWORKS}

In the analyses and examples in the previous sections of this paper, the number of neurons in the first layer 


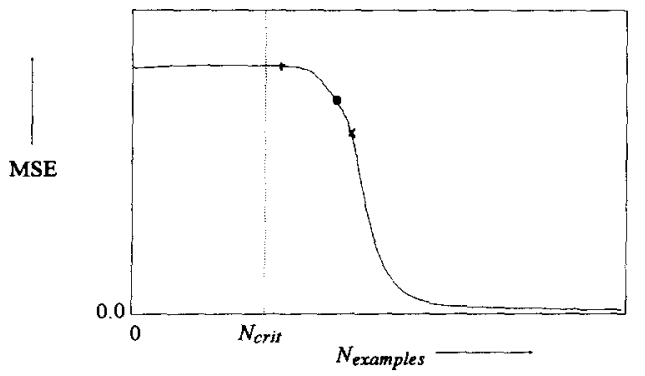

FIGURE 11. Simulated MSE for the XOR-problem; markers corresponding to the markers in the phase portrait in Figure 9.

was limited to two. It will be shown by induction that the analyses of Sections 4, 5, and 7 are also valid for neural nets with more than two neurons in the first layer.

For elucidation, assume a first layer with an arbitrary number of neurons. Assuming sufficiently small initial weights, the network can be linearized in the very beginning of training. During this phase, the weight vectors of all neurons will adapt almost identically, if again assuming only positive elements in $\underline{B}_{2,1}^{h}$. The starting conditions for the analyses in Sections 4, 5, and 7 will hence be satisfied in the very beginning of training for neural nets with an arbitrary number of neurons in the first layer.

As all neurons in the first layer adapt according to eqns (36), (42), and (60), it follows that all hyperplanes converge towards the same position in input space for $\tau \leq \tau_{\text {crit }}$ in eqns (56) or (63). At the end of this phase of training, the first layer will be highly redundant.

In the subsequent phase, for $\tau>\tau_{\text {crit }}$ in eqns (56) or (63), the cluster of redundant neurons effectively breaks into two clusters of redundant neurons, each cluster consisting of at least one neuron. This breaking agrees with the mechanisms derived for the two-neuron case in Sections 5 and 7. After eqn (30) is no longer satisfied, the two clusters classify a significantly different but neighboring part of the training set.

The learning behavior of each cluster after making a significantly different classification can be analyzed by making a new vector decomposition using the (new) attractor hyperplanes corresponding to the analyzed cluster. For each cluster, the analyses as presented in Sections 5 and 7 hold when neglecting the effects of other clusters on the analyzed cluster. Note that only neglecting the effect of neighboring clusters introduces a small error because only a small part of the examples classified by neighboring clusters is also classified by the analyzed cluster. It can be concluded that for networks with an arbitrary number of neurons in the first layer, the network builds up redundancy in the beginning of training, and iteratively abolishes the redundancy partially during further training by iteratively breaking of clusters of redundant neurons into smaller clusters.
An example of the learning behavior of a neural network with more than two neurons in the first layer is presented in Figure 12. Different phases in learning are represented by line segments between the shaded dots; training starts at the leftmost dot and proceeds towards the right.

In phase 1 , the weights are small enough to allow linearization and consequently redundancy in the first layer is built up; this phase has been discussed in Section 4.

In phase 2, linearization of the network is not allowed because neurons are generally activated outside the middle (approximately linear) region. In this phase of training, redundancy in the first layer is first increased and then gradually abolished by tending to subdivide the cluster of neurons into two smaller clusters. This subdivision can be either rotation-based (as presented in Section 5) or translation-based (presented in Section 7). The slow process of tending to break into multiple clusters may be recognized in the MSE versus training time curve as a temporary minimum.

In phases $3 a$ and $3 b$, the mutual redundancy is partially vanished; there are two clusters of neurons that classify a significantly different part of the input space. For both clusters, a new attractor hyperplane must be defined that corresponds to the subdivided training set. Strong redundancy exists in each cluster and the analysis can now be done for each cluster separately using the new attractor hyperplanes. As illustrated by the line segments $3 \mathrm{~b} 1$ and $3 \mathrm{~b} 2$, clusters may iteratively break into smaller clusters.

\subsection{Discussion of Very Small Initial Weights}

For the analysis presented in this paper, very small initial weights have been assumed. In neural network training, this assumption is generally satisfied when no a priori knowledge has been included in the weights. It has been shown in the previous subsection that for an appropriate training set (not separable by clustered hyperplanes in the first layer while better separable after breaking the cluster) the condition of very small initial

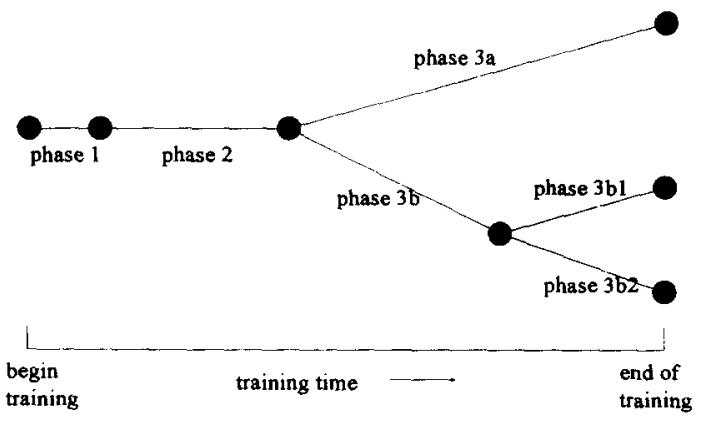

FIGURE 12. Example of the phases of the learning behavior of a neural network with more than two neurons in the first layer. 
weights leads to the encounter and escape of temporary minima.

However, if the initial weights are not very small according to the definition in Section 4, another mechanism leading towards temporary minima may occur. Although a thorough analysis of this mechanism is beyond the scope of this paper, this situation is discussed briefly.

It has been shown that under condition of very small initial weights, the redundancy is build up by clustering hyperplanes. If the condition of very small weights is not fulfilled, temporary minima may result from two types of redundancy. Firstly, one of the two clustering mechanisms derived for the case of very small initial weights may be present; phase $3 \mathrm{~b}$ in Figure 12 corresponds to a situation in which not small initial weights (initial here means at the beginning of the analysis of phase $3 b$ ) leads towards a temporary minimum due to clustering and breaking of the cluster.

Secondly, the neural network may only significantly adapt the weight vector of a part of the neurons in the first layer. This means that other neurons in the first layer are either not actively used in the classification or are trained such that they have a constant output that will act as an extra bias input for the neuron in the second layer. In the first case, the norm of the weight vector of these neurons initially remains relatively small and the associated weight in $\underline{W}_{2,1}$ also remains small. To escape an encountered minimum, a not-actively used neuron may become activated. In the latter case, the neurons have a constant response, resulting from positioning the hyperplane relatively far away from the training set while the norm of the weight vector corresponding to the hyperplane is large; these neurons will not actively be used in the classification.

\section{CONCLUSIONS}

Vector decomposition has been used for the mathematical analysis of the dynamics during training of twolayer feedforward neural networks learning according to the back-propagation algorithm. The utility of this novel analysis has been demonstrated by describing and explaining the so-called temporary minima that occur in two-layer network training.

Temporary minima are usually recognized from the MSE versus training time curve as a part in which the MSE is approximately constant for a long time after initial training. Better understanding of the mechanism behind this type of minimum is important because this may lead to finding methods for minimizing training time of two-layer neural nets.

It has been shown in this paper that the temporary minima result from subsequently building up redundancy in the first layer and removal of this redundancy. Removing the redundancy requires a relatively long training time in which the MSE sticks at a level cor- responding to a network with redundant neurons. As soon as the redundancy is (partially) abolished, the MSE curve drops relatively steep to a significant lower level corresponding to a less redundant neural network.

\section{REFERENCES}

Akaho, S. \& Amari, S. (1990). On the capacity of three-layer networks. Proceedings of International Joint Conference on Neural Networks, 2, 1-6.

Arai, M. (1989). Mapping abilities of three-layer neural networks. Proceedings of International Joint Conference on Neural Networks, 1, 419-423.

Gately, M. T. \& Penz, P. A. ( 1990). A geometrical overview of neural networks. TI Technical Journal, 7, 4-15.

Guckenheimer, J. \& Holmes, P. (1983). Nonlinear oscillations, dynamical systems, and bifurcations of vector fields. New York: Springer-Verlag.

Guo, H. \& Gelfand, S. B. (1991). Analysis of gradient descent learning algorithms for multilayer feedforward neural networks. IEEE Transactions on Circuits and Systems, 38, 883-894.

Hanson, S. J. \& Burr, D. J. (1988). Minkowski-r back-propagation: Learning connectionist models with non-Euclidian error signals. In D. Z. Anderson (ed.), Neural information processing systems (pp. 348-357). New York: AIP.

Heskes, T. M., Slijpen, E. T. P., \& Kappen, B. (1992). Learning in neural networks with local minima. Physical Review A, 46, 52215231.

Hirose, Y., Yamashita, K., \& Hijiya, S. (1991). Back-propagation algorithm which varies the number of hidden units. Neural Networks, 4, 61-66.

Hornik, K., Stinchcombe, M., \& White, H. (1990). Universal approximation of an unknown mapping and its derivatives using multilayer feedforward networks. Neural Networks, 3, 551-560.

Huang, S. C. \& Huang, Y. F. (1991). Bounds on the number of hidden neurons in multilayer perceptrons. IEEE Transactions on Neural Networks, 2, 47-55.

Irie, B. \& Miyake, S. (1988). Capabilities of three-layered perceptrons. Proceedings of International Joint Conference on Neural Networks, 1, 641-648.

Liang, P. (1991). Design artificial neural networks based on the principle of divide-and-conquer. Proceedings of IEEE International Conference on Circuits and Systems, 1319-1322. Singapore.

Lippmann, R. P. (1987). An introduction into computing with neural nets. IEEE ASSP Magazine, 4, 4-22.

Mehrotra, K. G., Mohan, C. K., \& Ranka, S. (1991). Bounds on the number of samples needed for neural learning. IEEE Transactions on Neural Networks, 2, 548-558.

Minsky, M. L. \& Papert, S. A. (1988). Perceptrons, an introduction to computational geometry (expanded edition). Cambridge, MA: MIT Press.

Murray, A. F. (1991a). Analog VLSI and multi-layer perceptronsAccuracy, noise, and on-chip learning. Proceeding of second International Conference on microelectronics for Neural Networks, 27-34. Munich: Kyrill \& Method Verlag.

Murray, A. F. (1991b). Analogue noise-enhanced learning in neural network circuits. Electronic Letters, 27, 1546-1548.

Parker, D. B. (1985). Learning logic (Tech. Rep. 47) Cambridge: MIT.

Rumelhart, D. E., Hinton, G. E., \& Williams, R. J. (1986). Learning internal representations by error propagation. Parallel distributed processing: Exploration in the microstructure of cognition (Vol. 1). Cambridge, MA: MIT Press.

Sheu, B. J. (1991). VLSI neurocomputing with analog programmable chips and digital systolic array chips. Proceeding of IEEE International Symposium on Circuits and Systems, 1267-1270. Singapore. 
Sontag, E. D. \& Sussman, H. J. (1991). Backpropagation separates where perceptrons do. Neural Networks, 4, 243-249.

Sussmann, H. J. (1992). Uniqueness of the weights for minimal feedforward nets with a given input-output map. Neural Networks, 5, 589-593.

Werbos, P. J. (1988). Backpropagation: Past and future. Proceedings of International Joint Conference on Neural Networks, 1, 343353.

Woods, D. (1988). Back and counter propagation abberations. Proceedings of International Joint Conference on Neural Networks, $1,473-479$.

Yang, H. \& Guest, C. C. ( 1990). Linear discriminants, logic functions, backpropagation, and improved convergence. Proceedings of International Joint Conference on Neural Networks, 3, 287-292.

\section{NOMENCLATURE}

$\underline{B}_{k, j}^{\text {bias }} \quad$ unit vector corresponding to only the bias input of the $j^{\text {th }}$ neuron in the $k^{t h}$ layer

$\underline{B}_{k, j}^{h} \quad$ unit vector perpendicular to the attractor hyperplane of the $j^{\text {th }}$ neuron in the $k^{t h}$ layer

dist distance between two hyperplanes corresponding to neurons in the first layer (two neuron in the first layer assumed); to be used for translation-based breaking

$E \quad$ energy function to be minimized during training

$M S E \quad$ mean squared error of the neural network

$M S E_{\min , r}$ minimum mean squared error of the neural network with redundancy

$M S E_{\min , n r}$ minimum mean squared error of the neural network without redundancy

$N_{\text {crit }} \quad$ number of examples passed during training until $\beta_{c r i t}$ is reached

$t \quad$ training time

$\underline{U}_{k} \quad$ input vector for the $k^{t h}$ layer including the bias input signal

$\underline{U}_{k, j}^{F} \quad$ input vector component of the $j^{\text {th }}$ neuron in the $k^{t h}$ layer in parallel to the associated attractor hyperplane.
$\underline{W}_{k j} \quad$ weight vector of the $j^{t h}$ neuron in the $k^{t h}$ layer

$W_{k, j}^{A T T} \quad$ weight vector corresponding to the socalled attractor hyperplane of the $j^{t h}$ neuron in the $k^{\text {th }}$ layer

$\underline{W}_{k, j}^{c} \quad$ weight vector component of the $j^{\text {th }}$ neuron in the $k^{t h}$ layer in parallel to the associated attractor hyperplane.

$Y_{k, j} \quad$ output of the $j^{t h}$ neuron in the $k^{t h}$ layer

$Y_{n e t} \quad$ output of the (single output) network

$\alpha_{k, j}^{\text {bias }} \quad$ value of the bias element in the input vector of the $k^{\text {th }}$ layer (identical for all $j$ )

$\alpha_{k, j}^{h} \quad$ norm of the input vector of the $k^{t h}$ layer perpendicular to the attractor hyperplane of the $j^{\text {th }}$ neuron in the $k^{\text {th }}$ layer

$\beta_{\text {crit }} \quad$ value of $\beta_{k, j}^{h}$ for two initially redundant neurons for which redundancy starts to be lifted

$\beta_{\max } \quad$ value of $\beta_{k, j}^{h}$ for two initially redundant neurons after an infinite training in which the redundancy is not lifted

$\beta_{k, j}^{\text {bias }} \quad$ value of the weight connected to the bias input of the $j^{\text {th }}$ neuron in the $k^{\text {th }}$ layer

$\beta_{k, j}^{h} \quad$ norm of the weight vector of the $j^{\text {th }}$ neuron in the $k^{t h}$ layer perpendicular to the associated attractor hyperplane

$\beta_{\text {valid }} \quad$ value of $\beta_{k, j}^{h}$ for two initially redundant neurons for which the analysis conditions are marginally satisfied

$\varphi$

angle between two hyperplanes corresponding to neurons in the first layer (two neuron in the first layer assumed); to be used for rotation-based breaking

$\Lambda \beta_{1}^{\text {bias }}$ difference of two bias-related weights $\beta_{1, j}^{\text {bias }}$ (assuming two neurons in the first layer) to be used for translation-based breaking of clustered hyperplanes.

$\underline{\Lambda} \underline{W}^{\varepsilon}$ difference vector between two $W_{1, j}^{\varepsilon}$ vectors (assuming two neurons in the first layer) $\tau_{\text {crit }} \quad$ training time $t$ at which $\beta_{\text {crit }}$ is reached 